\title{
Digitally monitoring thermalism health and wellness effects - A Conceptual Model Proposal
}

\author{
José Martins ${ }^{1,2,3}$, Fernando Moreira ${ }^{4}$, Manuel Au-Yong-Oliveira ${ }^{5}$, \\ Ramiro Gonçalves ${ }^{1,3}$ and Frederico Branco ${ }^{3}$ \\ ${ }^{1}$ AquaValor - Centro de Valorização e Transferência de Tecnologia da Água, Chaves, Portugal \\ ${ }^{2}$ Instituto Politécnico de Bragança, Campus de Santa Apolónia, Bragança, Portugal \\ ${ }^{3}$ INESC TEC, Porto, Portugal \\ ${ }^{4}$ IJP, REMIT, University Portucalense \\ ${ }^{5}$ INESC TEC, GOVCOPP, Department of Economics, Management, Industrial Engineering \\ and Tourism, University of Aveiro, Portugal \\ jose.martins@aquavalor.pt, fmoreira@upt.pt, mao@ua.pt, \\ ramirodutad.pt, fbrancoeutad.pt
}

\begin{abstract}
As life expectancy grows and the population requirements for satisfactory health and wellness levels increases, there is a clear opportunity for the incorporation of alternative techniques and tools for promoting health and preventing disease. One of these tools, is the use of thermal water-based treatments, commonly known as thermalism, as tools to trigger patients' overall wellness. Despite the collective assumption of the effects of thermalism, there is little to no scientific evidence of these treatments, thus impairing the potential of this activity to become more widespread in society. Thus, with this paper, we present a conceptual model for a non-invasive sensing system based on wearables that can monitor a set of patients' biomarkers which will serve as the basis for the validation of the effects of thermalism. This system will also serve as a management and operation control tool for thermal SPA managers and technical directors.
\end{abstract}

Keywords: Thermalism; Wearables; Non-invasive Sensing System; Sensing Systems; Wellness.

\section{Introduction}

In recent years, as global population average life expectancy registered a constant tendency to grow, an increased number of potential health pathologies and concerns have arisen, thus adding extra pressure for enduring efforts to further advance novel approaches for ensuring population wellness, the promotion of health and the prevention of disease [1].

Despite being considered a challenge of extreme relevancy and which has thus been the focus of multiple stakeholders (e.g.: researchers, healthcare organizations, governmental entities, etc.), promoting health and, particularly, wellness to a given population as a means to prevent disease and live longer, it is clearly a challenge of significant wingspan to which there is still no clear answer [2]. 
By calling on Martins, et al. [3] and Lupton [4], one can acknowledge that by merging the previously mentioned efforts with Information and Communication Technologies (ICT) we will be drawing innovative and disruptive solutions that will serve the purpose of promoting health and wellness in a more effective manner.

As argued by Araujo, et al. [5], the physical-chemical properties of thermal waters make them a valuable therapeutical asset that has the ability to not only trigger the curing process, but also to foster patients' wellness. The use of thermal waters for health and wellness purposes, typically known as thermalism, has a historic timeline that goes back to the ancient Roman civilization and currently has a significant impact on the economic and social development of multiple (more rural) regions [6][7].

Even though thermalism is considered an extremely relevant option for those willing to promote their own wellness and health, little research has been made on the effects of thermal water-based treatments [8][9]. With this in mind, an effort has been made to develop a conceptual model of an information system that is capable of monitoring, in a non-invasive manner, a series of personal indicators (capable of demonstrating thermalism effects), and in parallel feature an information visualization platform that, drawing on self-service business analytics capabilities, is able to give its users the overall impacts that each thermalism treatment has on each pathology, thus allowing for a full perception of the real opportunities that might exist associated with thermal water usage.

\section{Non-Invasive and Wearable Health and Wellness Sensing Systems}

Since the dawn of medicine, it has been fundamental to collect and analyse data from patients to study the behaviour of certain diseases, to find a cure and even to monitor their health status during their hospital stay or during recovery/treatment outside the hospital environment. E.g., in order to study a disease that affects a specific region, it is necessary to obtain information from the affected patients and keep the entire population of that region under surveillance. From a conceptual perspective, public health surveillance refers to the collection, analysis and continuous dissemination of healthrelated data to provide information that can be used to monitor and improve the health and wellness of populations [10]. The data collected during the observation period can be used to estimate the magnitude of a problem (disease) and to identify risk groups. Through the continuous monitoring of these patients, it is possible to evaluate the effectiveness of treatments to combat the disease under study [11].

With the current development of technology, new ways of collecting all types of data have emerged as well as improved tools for their treatment and analysis, which significantly increased the amount of data available for research and analysis purposes. This brings with it an increase in the amount of information available which is very positive for studies and research [12]. 


\subsection{Data Collection Principles}

The increase in the amount of data available also increases the amount of redundant and unnecessary data, so it is necessary to follow a series of principles regarding the selection and manipulation of data strictly necessary for the problem under study [13].

Initially, the objectives of the problem must be precisely defined, in order to avoid redundant and/or unnecessary data. If there are measurement standards, it is possible to do an automatic cleaning, discarding of erroneous or inconsistent data and, at the same time, creating alerts for measurements that present "dangers" to the user. In order to be able to catalogue the data and create a "trustworthy" database, there must be a minimum quantity, so that a correct analysis can also be made and that truly reflects the situation under study.

When we are using data collection tools that are too complex, this can negatively affect the quantity and quality of the data, so we must keep these tools as simple and concise as possible. After all the data has been collected, it must be kept safe and periodic analyses must be carried out on the consistency and accuracy of the database. There should also be a system for the protection of the integrity of the database and for security against possible computer attacks, natural disasters, and other threats.

\subsection{Wearable Sensing Systems}

The concept of the Internet of Things (IoT) encompasses situations in which network connectivity and computing capacity expand to everyday objects, sensors and items, and exchange data without the need for "handling" by the user [14]. Of these devices, those that can be used as accessories or that can be worn are called wearables [15].

Wearable technology has benefited from advances in mobile technology; however, they remain extremely limited in terms of functionality when compared to smartphones. While wearables must follow several restrictions regarding the size, weight and comfort of the device during use, smartphones do not have these restrictions. However, wearables allow for the opportunity to collect physiological and kinesiological data on an ongoing basis, translating into self-knowledge for users. This knowledge can be especially useful in the areas of health and fitness, by obtaining data during physical activity. These can also be used for the autonomous monitoring and prevention of diseases such as stress and hypertension [16][17].

According to Metcalf, et al. [14], as incentives are lining up and value-based healthcare (health delivery model in which hospitals and doctors are paid based on the patient's health outcomes) increases, the future will be the IoT that joins wearable devices, applications and intelligent data analysis. This is so that the medical and the care teams get the right insights from the data, at the right time, and are not overwhelmed. Smart preventive decisions can be made for more proactive individualized care and therapy.

Wearables can measure physiological signals such as electrocardiogram (ECG), heart rate (BPM), body temperature, electrodermal activity (property of the human body that causes continuous variation in the electrical characteristics of the skin), respiration rate, among many other signals. The continuous monitoring of these signals can help in the detection and diagnosis of various diseases, whether cardiovascular, 
neurological, or pulmonary in an early form. In addition, monitoring the individual's activities in real time, allows for the detecting of falls, posture analysis, or even an assessment of the hours of sleep [18].

Non-invasive sensors are suitable for the human user because they can take painless measurements and have no risk of infection. However, they are complex from the concept and design point of view. The choice of the system's location has limitations because it must obtain a good ratio between signal, noise, fixation, ergonomics, and discretion [19].

In order not to negatively affect the user's daily routine, the wearable system must be comfortable, have flexible components, reduced dimensions, be chemically inert, non-toxic, and hypoallergenic. To obtain a greater acceptance by the possible users of the system, it must be accessible, easy to use, non-intrusive and interoperable between the various computing platforms. Increasing the research and development of this type of systems, can improve the ease of use [20].

The choice of sensors is extremely important as they must be able to collect/read data accurately. They must also be able to connect to the processing unit so that data can be transmitted [21].

\section{The Evolution of Health and Wellness}

Since early times, human beings have been using thermal waters in a medicinal manner as a means to fight diseases, improve overall health conditions, extend life expectancies, and increase wellness levels [6]. In fact, inseparable from the concept of wellbeing, health is revealed as one of the unavoidable themes of the new millennium, and the tendency for people to have an increasing concern regarding their bodies and physical fitness, translates into the need to feel healthier, and even younger, in a permanent search for a better quality of life [22].

The concept of health has effectively evolved, and is no longer considered as the absence of disease (an empty notion of health), but rather as a life objective that, according to the World Health Organization (WHO) translates into a "state of complete physical, mental and social wellness" and not just, as was mentioned, the absence of disease or illness [23]. On the other hand, the Global Wellness Institute, stresses that the state of our health results from complex interactions between genetic factors and interrelated external variables, such as socioeconomic factors, the physical environment where we live and access to health care. These external factors form a complex "wellness ecosystem", which can influence, increasing or reducing, the impacts of genetic factors [24].

We are, therefore, facing a new health paradigm, based on new societal configurations, centred on a self-concern for well-being, on self-esteem, on the search for happiness, on personal and bodily fulfilment, on an increased sensitivity towards the risks and greater demand for services and health care that are aligned with a paradigm based on disease prevention and the promotion of balanced lifestyles, less focused on curing the disease and more focused on the active role and responsibility that citizens themselves can have in relation to their health [1]. 
According to the World Tourism Organization, thermalism will observe sustained growth over the next 20 years, mainly due to the emergence of new consumption patterns and motivations. Assuming the importance that thermal activity has for the territories where it is available, typically low-density territories and with low economic development rates, thermalism and the inherent tourist activity are decisive elements. In line with this reality are, for example, the regions of Alto Tâmega (in Portugal) and Galicia (in Spain) that have in their natural mineral waters (thermal and bottled) a solid pillar of tourist and economic development, as well as one of the strongest reasons for differentiation [25].

Although, historically, the practice of thermal treatments is associated with an improvement in the living conditions of users, this association is not sufficiently proven from a scientific point of view. Therefore, in the light of our knowledge, there is no exact scientific validation of the effects of thermalism, which makes the task of doctors even more difficult regarding the prescription of thermal water-based prescriptions.

\section{An Architecture for Monitor Thermal Treatment Effects}

The growing interest in the health sector on the one hand and on the other hand in the activities of SPAs, justify the attention given to the topic of incorporating information systems and technologies that allow not only the interoperability of data and information (decisive decision-making), but also to encourage the establishment of close and loyal relationships. In fact, we are talking about a sector of activity that, in recent years, has shown marked growth rates and in which any technological advances have direct implications for the quality of the services provided and, consequently, for the quality of life of citizens.

In Figure 1, we present the proposed architecture to monitor the effects of thermal water-based treatments on thermalists. Its description is set forth in this section.

\subsection{Interface Layer}

The Interface Layer aims to provide a set of applications that support daily management activities, at the strategic, tactical and operational levels. Operations Management is permanently focused on improving services, aiming to increase their quality while guaranteeing an alignment with the needs of strict cost control. The creation and development of services and their efficient management make up most of the activities inherent to this functional component. Operations personnel are responsible for ensuring that employees responsible for applying treatments have access to the necessary resources to be successful and, cumulatively, allow the organization to compete effectively in a highly competitive market.

Data Visualization is critical for building dashboards rich in Key Performance Indicators (KPI), aligned with business metrics to bring the organization's data to life through graphical representations that, desirably, provide a 360-degree view of the data, updated in real time and available on any device (e.g., tablet, mobile phone, or computer), and the more intuitive the report, the faster the decisions can be made. 
Fig. 1. Architecture for Monitoring Thermal Treatment
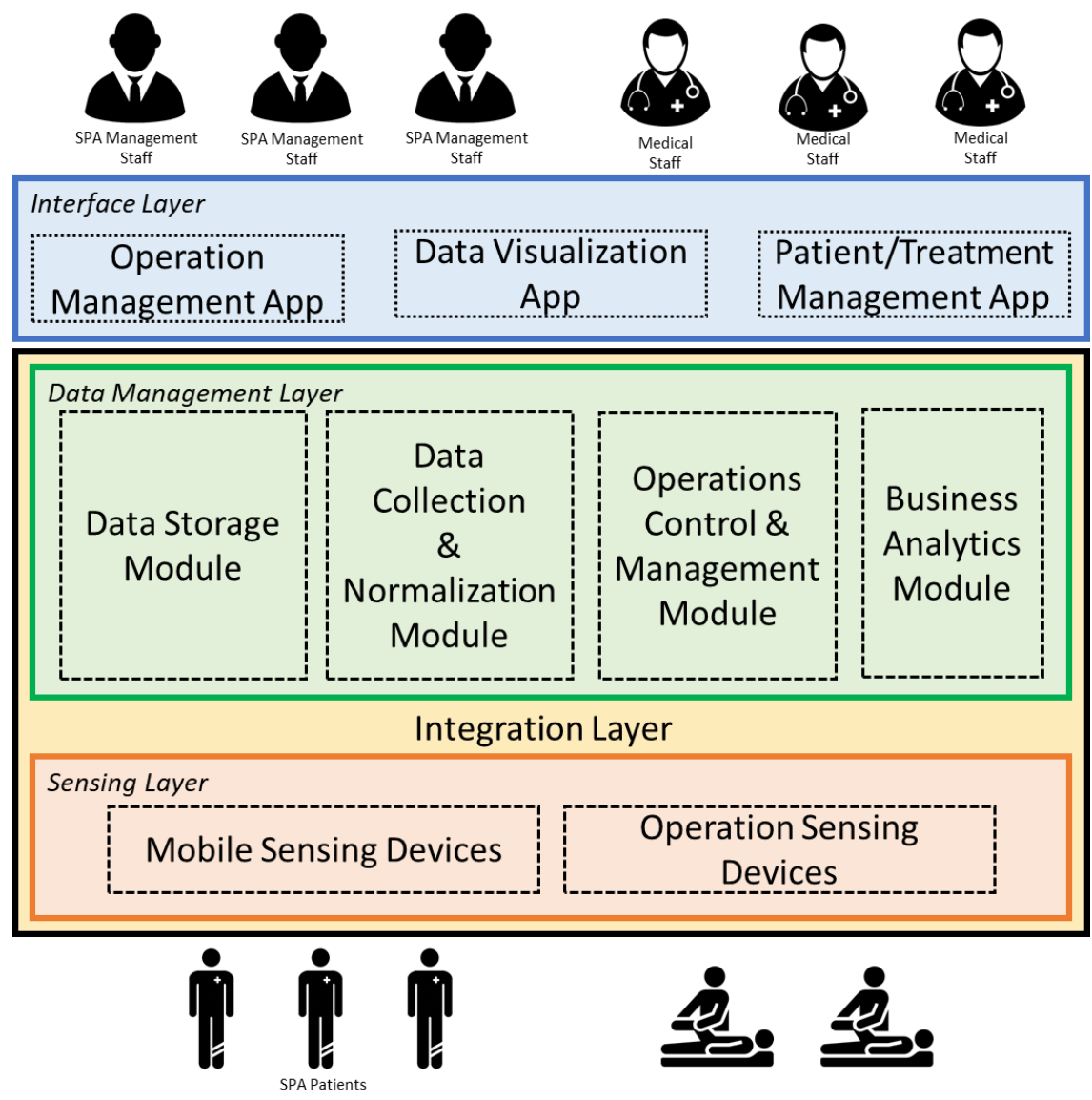

In order to ensure that both the medical and the technical teams have access to valid and accurate data that allows them to be aligned with each other, a Patient/Treatment Management App has been defined. Here, medical doctors and thermalism technicians can have access and manipulate patients' data (health, wellness and operational), and make decisions based on it. This component represents the main point of interface of the previously referred profiles with the information system.

\subsection{Data Management Layer}

The key tool of any Information System is data. The data can be considered as raw stored information, or in a more basic form. The reason why information is stored in this way is that only the information that is needed for specific functions is effectively used. The data represent elementary descriptions of facts, objects, activities or transactions that are recorded, classified and subsequently saved. When these data are orga- 
nized to provide meaning and value, it is information. Information thus plays an especially important role in information systems, as it is through it that the organization's operations are based. It is also important that there is no information overload or a database with too much information, as this can lead the organization to misinformation. The decisions that are made within an organization are based on a set of information that is available in the decision-making process, something that makes the final decision dependent on the characteristics of the information that the decision maker received. Information whose quality is insufficient will not produce an adequate decision, and when applied in the organization, it will not produce the expected result.

This makes the Data Storage module and the Data Collection \& Normalization modules critical elements for the management of data supporting the upper layer and, in particular, the Operations Control \& Management and Business Analytics modules. Massive data collection requires the use of adequate data storage and transformation technology, since exponential data growth has a direct relationship in terms of redundancy and deviations from acceptable value ranges, so it is necessary to follow a series of principles with regard to the selection of data strictly necessary to meet the analysis needs.

Therefore, it is critical in the Operations Control \& Management module to define the objectives of the problem precisely, to avoid redundant and/or unnecessary data. If there are measurement standards, it is possible to do an automatic cleaning and discarding of erroneous or inconsistent data while, at the same time, creating alerts for measurements that represent a "danger". In order to be able to catalogue the data and create a "trustworthy" database, there must be a minimum quantity, so that a correct analysis can also be made and one that truly reflects the situation under study.

When we are using data collection tools that are too complex, this can negatively affect the quantity and quality of the data, so we must keep these tools as simple and concise as possible. After all the data has been collected, it must be kept safe and a periodic analysis must be carried out on the consistency and accuracy of the database. There should also be a system for the protection of the integrity of the database and for security against possible computer attacks, natural disasters and other threats.

An important issue is the ability for different actors in organizations to make decisions based on quality data. The Business Analytics (BA) module aims to provide the orchestration of business concepts that will support the various players in the organization in making better decisions based on data and evidence. Decision-making will be more supported on certainties and done with tranquility and based on real and accurate data for organizational processes. The module should combine a large set of data analysis technologies, which allow for ad hoc analysis and consultation, online analytical processing (OLAP), mobile BA, real-time BA, operational BA, cloud BA and Software as a Service (SaaS), open-source BA and collaborative BA.

\subsection{Sensing Layer}

Regarding innovation accelerating technologies, the Internet of Things (IoT) offers a unique opportunity to transform many industries, including the thermalism and spa sector. The IoT comprises the expansion to a diversified range of objects and sensors with 
computing capacity and network connectivity without the need for user intervention in an extended period of use. Of these devices, those that can be used as accessories or that can be worn, are called wearables. In the IoT it is possible to include sensors, actuators, navigation systems, cloud-based data services, among others, that provide a wide variety of data to "feed" decision support tools.

The Mobile Sensing Devices component is essentially based on the use of wearables. Non-invasive sensors are suitable for the human user because they can make painless measurements and have no risk of infection. However, they are complex from the concept and design point of view. The choice of the system's location has limitations because it must obtain a good ratio between signal, noise, fixation, ergonomics and discretion.

In order not to negatively affect the user's daily routine, the wearable system must be comfortable, have flexible components, reduced dimensions, be chemically inert, non-toxic and hypoallergenic. In order to obtain greater acceptance by the possible users of the system, it must be accessible, easy to use, non-intrusive and interoperable between the various computing platforms.

The choice of sensors is extremely important as they must be able to collect/read data accurately. They must also be able to connect to the processing unit so that data can be transmitted. The low cost of these sensors can facilitate their adoption and massification (to eventually become mainstream).

\section{Conclusions}

\subsection{Limitations}

This paper represents the initial results of a research project focused on validating the health and wellness effects of thermalism, and on delivering digital solutions that allow for thermal SPA and healthcare managers to perceive the overall value and impact of thermalism to their organizations and regional/national healthcare systems.

Despite arising from a focused and extensive analysis of the existing scientific and technical literature that was merged with the knowledge and experience of healthcare and wellness experts and thermal SPA managers and technical directors, to create the proposed conceptual model, for a monitoring system that is able to monitor the effects of thermalism, to be considered a fully validated artifact it needs to serve as the basis for a real (Information) System based on wearable devices which allow for non-invasive monitoring of wellness and health biomarkers on their users.

\subsection{Future Implications for Thermalism}

As argued by both academics and healthcare and wellness experts, thermalism is globally perceived as a valuable treatment or adjuvant for the symptoms associated with a set of impairments and diseases. However, the real effects of thermal water-based treatments on patients are yet to be scientifically proven, which diminishes the potential impact that thermalism might have on patients' lives and regarding the social and economic development of the regions where the referred natural resources exist. 
The implementation of the proposed conceptual model might allow for the appearance of technology-based solutions that are not only able to monitor the health and wellness effects of thermalism, but also to acknowledge the social and economic impacts that this activity has on regional development and on national health systems.

The emergence of digital systems and platforms that allow a validation of the effects of thermalism, a consequent evolution of this activity and, ultimately, a change to a healthier lifestyle on the part of citizens, is something that should be considered as remarkably interesting and relevant. Considering the current health and well-being needs, the referred evolution may not only benefit the thermal spa (currently in Portugal, for example, there are already more than $1.2 \mathrm{M}$ thermal treatments per year), but also spa and national health services, since the inclusion of thermal treatments (duly validated) in national plans for disease prevention and health promotion may lead to a decrease in the effort required to provide health care and well-being to the entire population.

Another potential future implication of implementing systems that validate the effects of thermalism and, consequently, allow the development of thermal activity and the thermal SPAs themselves, is the attraction and fixation of people to the regions where these services exist. From a social and economic point of view, these territories are typically rural and considered to be of extremely low development and low population density. Therefore, the population fixation, typically highly specialized, focused on the provision of thermal treatments, the development of systems and technological platforms related to thermalism, and the provision of services adjacent to thermalism (e.g., tourism), can serve as an engine for territorial growth and development.

\subsection{Conclusions}

Thermalism is currently considered one of the most valuable assets for ensuring relevant wellness levels to a fringe of the population typically known for having physical impairments or issues. Nevertheless, despite this social perception, little to no scientific evidence has been reached nor presented that could validate the real effects of thermalism on patients' impairments or health/wellness issues.

By acknowledging the abovementioned argument, an effort has been made to model an information system that should be able to, not only monitor the individual effects that thermal water-based treatments have on patients' pathologies, but also to assist thermal SPA managers and technical directors to better manage their organizations and improve the services they provide for their patients. This conceptual model proposal contemplates three combined layers (Sensing, Data Management, and Interface) that are composed of multiple functional modules responsible for the execution of the entire set of functional requirements.

By providing a straightforward approach, drawn on the incorporation of ICT, to the validation of the effects of thermalism, we believe that we are not only delivering a valid contribution to science, but that we are also creating tools that will trigger (regional) social and economic development. 


\section{Acknowledgments}

This work is financed by National Funds through the Portuguese funding agency, FCT - Fundação para a Ciência e a Tecnologia within project UIDB/50014/2020.”

\section{References}

1. Gonçalves, E.C., Guerra, R.J.d.C.: O turismo de saúde e bem-estar como fator de desenvolvimento local: uma análise à oferta termal portuguesa. (2019)

2. Leandro, M.E., Leandro, A.S.d.S.: Da saúde e bem-estar/mal-estar ao termalismo. Sociologia 30, 75-96 (2015)

3. Martins, J., Gonçalves, R., Branco, F.: A full scope web accessibility evaluation procedure proposal based on Iberian eHealth accessibility compliance. Computers in Human Behavior 73, 676-684 (2017)

4. Lupton, D.: Digital health now and in the future: Findings from a participatory design stakeholder workshop. Digital health 3, 2055207617740018 (2017)

5. Araujo, A.R., Paiva, T., Ribeiro, M.P., Coutinho, P.: Innovation in Thermalism: An Example in Beira Interior Region of Portugal. Health and Wellness Tourism, pp. 165-180. Springer (2015)

6. Maldonado-Erazo, C.P., Álvarez-García, J.: State of the art of research in the sector of thermalism, thalassotherapy and spa: A bibliometric analysis. European Journal of Tourism Research 19, (2018)

7. Costa, C., Quintela, J., Mendes, J.: Health and wellness tourism: A strategic plan for tourism and thermalism valorization of São Pedro do Sul. Health and wellness tourism, pp. 21-31. Springer (2015)

8. Cacciapuoti, S., Luciano, M.A., Megna, M., Annunziata, M.C., Napolitano, M., Patruno, C., Scala, E., Colicchio, R., Pagliuca, C., Salvatore, P.: The Role of Thermal Water in Chronic Skin Diseases Management: A Review of the Literature. Journal of Clinical Medicine 9, 3047 (2020)

9. Ruela, L.d.O., Moura, C.d.C., Gradim, C.V.C., Stefanello, J., Iunes, D.H., Prado, R.R.d.: Implementation, access and use of integrative and complementary practices in the unified health system: a literature review. Ciencia Saude Coletiva 24, 4239-4250 (2019)

10. Soucie, J.M.: Public health surveillance and data collection: general principles and impact on hemophilia care. Hematology 17, s144-s146 (2012)

11. Dormann, H., Eder, P.A., Gimpel, H., Meindl, O., Rashid, A., Regal, C.: Assessing healthcare service quality using routinely collected data: Linking information systems in emergency care. Journal of Medical Systems 44, (2020)

12. Santosh, K.: AI-driven tools for coronavirus outbreak: need of active learning and crosspopulation train/test models on multitudinal/multimodal data. Journal of medical systems 44, 1-5 (2020)

13. Schneble, C.O., Elger, B.S., Shaw, D.M.: All our data will be health data one day: the need for universal data protection and comprehensive consent. Journal of Medical Internet Research 22, e16879 (2020) 
14. Metcalf, D., Milliard, S.T., Gomez, M., Schwartz, M.: Wearables and the internet of things for health: Wearable, interconnected devices promise more efficient and comprehensive health care. IEEE pulse 7, 35-39 (2016)

15. Niknejad, N., Ismail, W.B., Mardani, A., Liao, H., Ghani, I.: A comprehensive overview of smart wearables: The state of the art literature, recent advances, and future challenges. Engineering Applications of Artificial Intelligence 90, 103529 (2020)

16. Dunn, J., Runge, R., Snyder, M.: Wearables and the medical revolution. Personalized medicine 15, 429-448 (2018)

17. Liu, C., Liu, F., Zhang, L., Su, Y., Murray, A.: Smart Wearables in Healthcare: Signal Processing, Device Development, and Clinical Applications. Journal of healthcare engineering 2018, (2018)

18. Park, S., Chung, K., Jayaraman, S.: Wearables: Fundamentals, advancements, and a roadmap for the future. Wearable sensors, pp. 1-23. Elsevier (2014)

19. Lmberis, A., Dittmar, A.: Advanced wearable health systems and applications-research and development efforts in the European union. IEEE Engineering in Medicine Biology Magazine 26, 29-33 (2007)

20. Majumder, S., Mondal, T., Deen, M.J.: Wearable sensors for remote health monitoring. Sensors 17, 130 (2017)

21. Gupta, M.S.D., Patchava, V., Menezes, V.: Healthcare based on iot using raspberry pi. In: 2015 International Conference on Green Computing and Internet of Things (ICGCIoT), pp. 796-799. IEEE, (2015)

22. Ramos, A.R.: O novo paradigma dos destinos turisticos termais. Gestión turística 9-36 (2008)

23. WHO, Constitution of the World Health Organization, Available: https://www.who.int/about/who-we-are/constitution, Accessed: December 2020

24. GWI, Global Wellness Economy Monitor: Thermal/Mineral Springs, Available: www.globalwellnessinstitute.org, Accessed: October 2020

25. Pinos Navarrete, A., Shaw, G.: Spa tourism opportunities as strategic sector in aiding recovery from Covid-19: The Spanish model. Tourism Hospitality Research 1467358420970626 (2020) 